\title{
Fate and toxic effects of environmental stressors: environmental control
}

\author{
Jie Zhuang ${ }^{1,2} \cdot$ Han-Qing $\mathrm{Yu}^{3} \cdot$ Theodore B. Henry ${ }^{4,5} \cdot$ Gary S. Sayler $^{5}$
}

Accepted: 8 October 2015/Published online: 24 October 2015

(C) Springer Science+Business Media New York 2015

\begin{abstract}
The potential for toxicants to harm organisms in the environment is influenced by the physicochemistry of the substances and their environmental behaviors and transformation within ecosystems. This special issue is composed of 20 papers that report on studies which have investigated the fate and toxicity of various toxicants including engineered nanoparticles, pharmaceuticals and personal care products, antibiotics, pathogens, heavy metals, and agricultural nutrients. The environmental transformations of these substances and how these processes affect their toxicity are emphasized. This paper highlights the important findings and perspectives of the selected papers in this special edition, with an aim of providing insights into full-scale evaluation on the toxicity of various contaminants that exist in ecosystems. General suggestions are provided for the future directions of toxicological research.
\end{abstract}

Jie Zhuang

zhuangjie@iae.ac.cn; jzhuang@utk.edu

1 Key Laboratory of Pollution Ecology and Environmental Engineering, Institute of Applied Ecology, Chinese Academy of Sciences, Shenyang 110016, China

2 Department of Biosystems Engineering and Soil Science, The University of Tennessee, Knoxville, TN 37996, USA

3 School of Chemistry and Materials, University of Science and Technology of China, Hefei, China

4 School of Life Sciences, Heriot-Watt University, John Muir Building, Edinburgh EH14 4AS, UK

5 Department of Microbiology, Center for Environmental Biotechnology, The University of Tennessee, Knoxville, TN 37996, USA
Keywords Nanoparticles - Toxicity - Nutrient - Heavy metals $\cdot$ Pathogen $\cdot$ PPCP

\section{Introduction}

Anthropogenic activities, such as agricultural production, urban development, and application of novel chemicals and engineering materials, have released a huge volume of harmful materials to the environment that have damaged ecosystem functions and threatened human and wild life health (UNEP 2013). In addition, reutilization of resources (e.g., wastewater and sludge) and energy production (e.g., shale gas, biofuels, and hydrogen) produce toxic materials and create toxicological challenges. The possibility of that contaminants with toxic properties occur as a consequence of nearly all human activities is a threat that is well recognized, and addressing this issue is a high priority topic for environmental scientists and regulatory agencies throughout the world. Thus far, numerous studies have been conducted to address the risks of various toxicants in the environment, but the relations between the fate and the toxicity of contaminants are not yet clearly defined due to the complexity of the environmental behavior of toxicants upon release into the environment and how this may influence toxicological processes. Integrated studies of toxicological processes are urgently needed to fully consider and quantify the contributions of each component of the environmental processes that affect toxicity of contaminant.

Given the current interest by those in the environmental control arena to understand the fate-toxicity relation of anthropogenic contaminants in ecosystems or production scenarios, the guest editors have assembled this special issue of Ecotoxicology. Papers were chosen to illustrate the 
advances in characterizing and evaluating the environmental behaviors and associated toxicity of various pollutants, including engineered nanoparticles, heavy metals, pharmaceuticals and personal care products (PPCPs), antibiotics, pathogens, and nutrients. This collection of 20 selected papers in this Special Issue represents a result of scientific exchange and research discussion among the scientists and students of the United States and China at the China-US Joint Symposium of Energy, Water, and Ecosystem Sustainable Development. The symposium was jointly organized by the US-China Ecopartnership for Environmental Sustainability and the China-US Joint Research Center for Ecosystem and Environmental Change and held on October 26-28, 2014, in Hefei, China, with the sponsorship of the Natural Science Foundation of China. More than eighty representatives from academia, government, and industry in China and the United States gathered to present and discuss their latest scientific findings and current challenges of toxicological risks encountered in food production, energy production, and human health.

All of the submitted papers for this special issue were rigorously peer reviewed in accordance with the policies of this journal. The articles accepted for publication cover a wide range of topics relating to the fate and toxic effects of various contaminants generated during wastewater treatment and application, energy production, drinking water supply, animal production, and agricultural and fishery production. To facilitate understanding of the complexity of environmental control to the fate and toxic effects of various stressors, the guest editors have grouped the papers into six common themes: nanoparticle toxicity, influence of wastewater treatment on fate and toxicity of toxicants, effect of disinfection on substance toxicity, toxicological challenges of renewable energy production, ecological effects of excessive nutrients, and heavy metal toxicology.

\section{Toxic effect of engineered nanoparticles}

The stability and bioavailability of engineered nanoparticles could be modified by environmental physicochemical conditions. As well, environmental factors, such as humic acid and solution chemistry, are important factors determining the fate and toxicity of nanomaterials in the environment. In general, environmental conditions have a large potential for mediating the toxicity of engineered nanoparticles. Du et al. (2015) evaluated the toxicity of cellulose nanocrystals (CNCs) by assessment of the response of constitutively bioluminescent luxCDABEbased bioreporter Escherichia coli 652T7. Results demonstrated that $\mathrm{CNCs}$ were not significantly toxic at concentrations at or below $250 \mathrm{mg} / \mathrm{L}$, but the toxicity increased linearly as $\mathrm{CNC}$ concentrations increased from 300 to $2000 \mathrm{mg} / \mathrm{L}$. Longer exposure time and elevated solubility of the CNCs were shown to increase cytotoxicity effects. Fang et al. (2015) studied the effects of humic acid and ionic strength on the sub-lethal toxicity of $\mathrm{TiO}_{2}$ nanoparticles to zebrafish by monitoring the changes of superoxide dismutase, catalase, malonaldehyde, and glutathione in gill, liver, and intestine. The nanoparticle aggregation was found not an essential factor to influence nanoparticle toxicity. However, humic acid and/or ionic strength decreased the bioavailability of $\mathrm{TiO}_{2}$ nanoparticles in water. Xiong et al. (2015) studied the effect of morphology of $\mathrm{Cu} / \mathrm{Cu}_{\mathrm{x}} \mathrm{O}$ nanoparticles on their antimicrobial activity. Their results show that the flower-like $\mathrm{Cu} / \mathrm{Cu}_{\mathrm{x}} \mathrm{O}$ nanoparticles were the most effective form to inhibit microbial growth. This morphology dependent effect was attributed to the difference in the surface free energy among different crystal facets of the copper nanoparticles.

\section{Fate and toxicity of contaminants during wastewater treatment and application}

Pharmaceuticals and personal care products (PPCPs) are often found in the effluents from wastewater treatment plants due to insufficient removal during wastewater treatment processes. Agricultural application of reclaimed water, sludge, and animal wastes has brought PPCPs into the natural environment (Qin et al. 2015a). Because of the biological activity of PPCPs even at low environmental concentrations, the potential risks of these substances to humans and organisms in ecosystems have caused great concern. However, little was known about their environmental behaviors and toxic effects. Chen et al. (2015) performed a study to assess the removal of frequently occurring pharmaceuticals (naproxen, fenoprofen, ketoprofen, dichlofenac, and carbamazepine) and the biocide triclosan in activated sludge from four different wastewater treatment plants in Demark. The most rapid degradation was observed for NSAID pharmaceuticals (naproxen, fenoprofen, and ketoprofen) at degradation rates of 46-94\%, while dichlofenac and carbamazepine were persistent in the wastewater treatment systems. Interestingly, no relationships were observed between degradation rates and biomass concentrations in the diverse sludge materials that were evaluated.

The presence of toxicants in sewage sludge impacts the potential for the sludge to be used as an amendment to soils. For example, the presence of nonylphenol in sewage sludge prevents the use of the sludge. Composting is one of the most efficient and economical methods of making sewage sludge stable and harmless. Zheng et al. (2015a) 
studied the nonylphenol degradation rates during composting under the conditions of adding bulking agents (sawdust, corn stalks, and mushroom residue) and applying aeration. Addition of $20 \%$ mushroom residue and $20 \%$ sawdust to the sewage sludge led to the highest $(71.6 \%)$ and lowest $(22.5 \%)$ apparent nonylphenol degradation rates, respectively. The study suggests that adding biomass materials and appropriate aerobic conditions could facilitate the degradation of nonylphenol in sewage sludge. To monitor the estrogenic, androgenic and toxic potencies in wastewater, Wang et al. (2015a) demonstrated a standardized protocol for bioluminescent strains Saccharomyces cerevisiae BLYES, BLYAS, and BLYR as highthroughput screening tools. The standardized protocol was applied for a year-long monitoring of the effluent of a wastewater treatment plant featuring parallel operated fullscale membrane bioreactor and traditional activated sludge treatment. Monitoring results showed that estrogenic activity was persistent in the effluent samples, while residual androgenic activity was not detected throughout the monitored period. Irrigation of agricultural lands can cause contamination of soils with pharmaceutical substances and risks to human and ecosystem health. Xing et al. (2015) summarized the progress of studies on the role of soil colloids in mediating the environmental behaviors of pharmaceuticals. Reported results showed that colloids can adsorb pharmaceuticals mainly through ion exchange, complexation, and non-electrostatic interactions. The adsorbed pharmaceuticals may have higher risks to induce antibiotic resistance; besides, their transport may also be altered considering they have great chance to move with colloids. Solution conditions (such as $\mathrm{pH}$, ionic strength, and cations) could influence these interactions between pharmaceuticals and colloids. Zeng et al. (2015) evaluated the health risk of six heavy metals $(\mathrm{Zn}, \mathrm{Cu}, \mathrm{Cr}, \mathrm{Ni}, \mathrm{Pb}$, and Cd) via dietary intake of wheat grown in a sewage irrigation area for a long history. Results showed that the continuous application of wastewater has led to an accumulation of heavy metals in the soil and in wheat. The health risk due to the effects of accumulated heavy metals (especially $\mathrm{Cd}$ and $\mathrm{Cr}$ ) was more significant for children than for adults.

\section{Toxic effect during disinfection process}

Toxic risks are associated with disinfection processes applied to animal and human wellbeing as well as drinking water production and supply. For instance, antibiotics have been widely used for disease prevention and treatment of the human and animals, and for growth promotion in animal husbandry. However, antibiotics can disturb the intestinal microbial community. Misuse or overuse of antibiotics can result in increase and spread of microbial antibiotic resistance, threatening human health and ecological safety. In this special collection, Yin et al. (2015) reported intestinal microbial community shift and antibiotic resistance alteration of the mice that drink the water containing tetracycline hydrochloride (TET). Metagenomic analysis showed that TET treatment affected a number of functions of the intestinal microorganisms and enhanced the abundance of antibiotic resistance genes by $\sim 5$ times, plasmids by $\sim 8$ times and integrons by $\sim 200$ times in mice gut. The research suggests that TET administration could increase the opportunity of antibiotic resistance genes and mobile genetic elements entering into the environment with feces discharge. E. coli $\mathrm{O} 157: \mathrm{H} 7$ is a significant human pathogen that is continually responsible for sickness and even death on a worldwide scale. Xu et al. (2015b) reported that a critical bacterial infection level (i.e., $5-10 \times 10^{5}$ ) must be reached prior to the onset of cytotoxic effects of E. coli O157:H7. They found that cells displaying infection-mediated metabolic activity reductions could recover to wild type metabolic activity levels if the infecting bacteria were removed prior to cell death. These results indicate that rapid treatment of $E$. coli O157:H7 infection could serve to limit host metabolic impact and reduce overall host cell death. As for drinking water production, Tian et al. (2015) reported an innovative and removable water treatment system for reducing waterborne pathogens, which consists of strong electric field discharge and hydrodynamic cavitation based on advanced oxidation technologies (AOT). The biological efficacy and toxicity effects of this AOT based system were evaluated during water disinfection treatments. Results showed that high inactivation efficiency of $E$. coli $(>5 \mathrm{log}$ ) could be obtained in synthetic contaminated water at low concentration $(0.5-0.7 \mathrm{mg} / \mathrm{L})$ of total oxidants in 3-10 s. Drinking water supply is mostly subject to chlorine stress. Free chlorine is a potent oxidizing agent and has been used extensively as a disinfectant in processes including water treatment. However, the excessive residual of free chlorine in water distribution systems might cause adverse health effects. As the first effort to assess the fate of chlorine in premise plumbing, Zheng et al. (2015b) investigated the performance of three types of actual premise plumbing pipe materials (i.e. copper, galvanized iron, and PVC) in maintaining free chlorine residual. The most rapid chlorine decay was observed in copper pipes, suggesting the lower toxic risk of chlorine and the need for higher chlorine dosage to maintain appropriate chlorine level if copper piping is used. PVC pipes exhibited the least reactivity with free chlorine, implying the higher toxic risk of chlorine and the advantage of PVC material for maintaining free chlorine residual. 


\section{Toxicological challenges associated with renewable energy production}

Toxicological challenges exist during renewable energy production because of the release of potentially cytotoxic or inhibitory compounds from input feedstocks, the metabolic co-synthesis of ethanol and potentially detrimental byproducts, and the potential cytotoxicity of ethanol itself. In recent years, a significant effort has been dedicated to mitigating the toxicological challenges encountered across the bioenergy production process. In this special edition, Akinosho et al. (2015) reviewed the major toxicological challenges encountered throughout each stage of the bioethanol production process and the commonly employed strategies for reducing or eliminating potential toxic effects. They suggest that each stage of bioethanol production should adopt genetic or engineering controls to ensure the health and productivity of the host organisms during a large scale production of bioethanol. In addition, the detoxification of resulting wastes becomes increasingly important as production levels increases. In addition, the need to stimulate microbial activities for contaminant degradation and energy generation has spurred intensive researches for the development of enhancement technologies. Microbial fuel cells have emerged as a promising technology for wastewater treatment coupled with clean energy production. Tong et al. (2015) demonstrates that a weak static magnetic field could be used as a simple and effective approach to stimulate microbial activities in sludge inoculated microbial fuel cells, and may offer implications for bioelectrochemical decontamination applications.

\section{Ecological effects of excessive nitrogen and phosphorus}

Anthropogenic activities (e.g., agricultural cultivation, wastewater release) have caused serious eutrophic environmental problems in many regions of the world. Biological responses to the change in growth conditions in the eutrophic environments continue to be a focused research in ecotoxicology. Relevant research include comprehensive evaluation of the roles of sediment in the restoration of vegetation in eutrophic waters, ecological risk assessment of nutrient pollution, and change in biodiversity and food web. In this special edition, $\mathrm{Wu}$ et al. (2015) investigated the effect of sediment anoxia on the acclimation mechanisms of a typical pollution-tolerant submerged macrophyte species (Hydrilla verticillata) in an eutrophic water environment. $H$. verticillata growth was significantly affected by highly anoxic conditions. However, slight anoxia $(0.1 \%$ sucrose addition) promoted growth. The increased anaerobic respiration and changes in carbon and nitrogen metabolism in roots show that $H$. verticillata has a degree of tolerance to anoxic conditions. To address the impact of agricultural nutrients on wetland ecosystem, Wang et al. (2015b) assessed nematode ecological indexes based on the soil properties of wetlands and adjacent farmlands. The results suggested that disturbance to wetland ecosystems by nitrogen and phosphorus reduced the richness of bacterivore nematodes. Nematode Hsp70 could be a useful biomarker to indicate the change in the levels of nitrogen and phosphorus concentrations in the studied wetlands.

\section{Bioavailability and toxicity of heavy metals}

The interactions between heavy metals and natural organic matter (NOM) substantially affects their migration and conversion in natural environments. In this special issue, $\mathrm{Xu}$ et al. (2015a) addressed the influence of organic ligands on the microbial availability of ionic mercury $\left(\mathrm{Hg}^{2+}\right)$ using a luxCDABE-based genetically engineered bacterial bioreporter (E. coli ARL1). Results showed that the E. coli ARL1 bioreporter was sensitive to $\mathrm{Hg}^{2+}$, with a detection limit of $\mathrm{Hg}^{2+}$ of $0.5 \mu \mathrm{g} / \mathrm{L}$ and an excellent dose-response correlation up to $2000 \mathrm{Hg}^{2+} \mu \mathrm{g} / \mathrm{L}$. Two organic ligands (humic acids and EDTA) reduced the bioavailability of $\mathrm{Hg}^{2+}$ via chemical complexation. The use of $E$. coli ARL1 is a costeffective, rapid, and reliable approach for measuring aqueous mercury at very low concentrations and thus has potential for applications in field in situ monitoring. Luo et al. (2015a) investigated the chemical speciation and element mapping of $\mathrm{Fe}$ and $\mathrm{Mn}$ under the conditions of reduced NOM. The results show that quinone and semiquinone moieties dominated the redox properties in NOM and that the NOM-Fe/Mn complexes had the characteristics of superparamagnetic oxyhydroxides. The results suggest that the surface interactions, which are closely related to the redox state of NOM could considerably influence the surface interactions of metals and in turn their speciation, immobilization, transport, and toxicity in natural environments.

In the biological uptake of heavy metals, Luo et al. (2015b) investigated the bioaccumulation of $\mathrm{Cd}$ in the gonads of tilapia via dissolved and dietary routes and maternal transfer of $\mathrm{Cd}$. The results showed that $\mathrm{Cd}$ was accumulated significantly in both ovary and testis from both exposure routes. The $\mathrm{Cd}$ exposure displayed different effects on gene expressions in gonads. Histopathological analysis showed that $\mathrm{Cd}$ induced injuries of ovary and testis. The results demonstrated that both dissolved and dietary $\mathrm{Cd}$ exposures affected gonad development by altering steroid hormone levels and sex-related gene expressions in tilapia. Qin et al. (2015b) studied the characteristics of $\mathrm{Cd}$ uptake by roots and translocation from 
roots to leaves of two eggplant species (Solanum melongena and Solanum torvum) under relatively low Cd concentrations using stable ${ }^{108} \mathrm{Cd}$ isotope through a number of hydroponic experiments. The results suggested that the phloem played a dominant role in the long-distance transport of Cd from the root to the leaf of S. melongena, whereas the xylem was the main channel for the translocation of $\mathrm{Zn}$ and ammonium ion.

\section{Summary and outlook}

The toxic effects of environmental stressors are subject to environmental conditions and process dynamics, and their degrees vary with the scales of associated processes or systems. The fate and stressors and their interactions with various environmental factors determine their bioavailability and final toxic effects. Therefore, a large potential exists for mediating the toxicity of inorganic, organic, and biological agents through environmental or biological manipulation. The current needs are to (1) thoroughly understand the behaviors of various stressors in each associated environment and their toxic effects at multiple scales, (2) establish long-term observation of stressor's toxic effect in respect to eco-environmental management practices, (3) create a theoretical framework to describe the fate-uptake-toxicity relations, and (4) develop self-restoring capability or adaptability of stressed systems by utilizing the interactions among involved factors or regulating the mechanisms governing the biological, chemical, and/or biological processes. In short, toxicological effects can be controlled if the fate of environmental stressors is well understood and this knowledge is used to manage the exposure of organism and ecosystems to toxicants.

Acknowledgments This special issue is the outcome of a Joint Symposium of Energy, Water, and Ecosystem Sustainable Development", which was held on October 26-28, 2014 in Hefei and organized by the US-China Ecopartnership for Environmental Sustainability and the China-US Joint Research Center for Ecosystem and Environmental Change. The workshop was jointly funded by the Natural Science Foundation of China (Grant Number: 21261160489) and the Strategic Priority Research Program of the Chinese Academy of Sciences (Grant Number: XDB14020204).

\section{Compliance with ethical standards}

Conflict of interest The authors declare they have no conflict of interest.

\section{References}

Akinosho H, Rydzak T, Borole A, Ragauskas A, Close D (2015) Toxicological challenges to microbial bioethanol production and strategies for improved tolerance. Ecotoxicology 24:1-19
Chen X-J, Vollertsen J, Nielsen JL, Gieraltowska A, Bester K (2015) Degradation of PPCPs in activated sludge from different WWTPs in Denmark. Ecotoxicology 24:1-8

Du L-Y, Arnholt K, Ripp SA, Sayler GS, Wang S-Q, Zhuang J (2015) Biological toxicity of cellulose nanocrystals (CNCs) against the luxCDABE-based bioluminescent bioreporter Escherichia coli 652T7. Ecotoxicology 24:1-5

Fang T, Yu L-P, Zhang W-C, Bao S-P (2015) Effects of humic acid and ions strength on $\mathrm{TiO}_{2}$ nanoparticles sublethal toxicity to zebrafish. Ecotoxicology 24:1-13

Luo H-W, Lin Z-Q, Yu H-Q, Sheng G-P (2015a) Spectroscopic characterization of the complexes between $\mathrm{Fe} / \mathrm{Mn}$ and natural organic matters by electron paramagnetic resonance and synchrotron-based techniques. Ecotoxicology 24:1-6

Luo Y-J, Shan D, Zhong H, Zhou Y, Chen W-Z, Cao J-L, Guo Z-B, Xiao J, Huang Y-F, Li J, Huang H-M, Xu P (2015b) Subchronic effects of cadmium on the gonads, expressions of steroid hormones and sex-related genes in tilapia Oreochromis niloticus. Ecotoxicology. doi:10.1007/s10646-015-1542-5

Qin Q, Chen X-J, Zhuang J (2015a) The fate and impact of pharmaceuticals and personal care products in agricultural soils irrigated with reclaimed water. Crit Rev in Env Sci Technol 45(13):1379-1408

Qin Q, Li X-M, Zhuang J, Weng L-P, Liu W, Tai P-D (2015b) Longdistance transport of cadmium from roots to leaves of Solanum melongena. Ecotoxicology 24:1-9

Tian Y-P, Yuan X-L, Li R-H, Xu S-J, Zhou X-Y, Zhang Z-T (2015) Biological efficacy and toxicity effect of emergency water disinfection process based on advanced oxidation technology. Ecotoxicology 24:1-10

Tong Z-H, Yu H-Q, Li W-W, Wang Y-K, Sun M, Liu X-W, Sheng G-P (2015) Application of a weak magnetic field to improve microbial fuel cell performance. Ecotoxicology 24:1-6

United Nations Environment Programme (UNEP) (2013) Report on the costs of inaction on the sound management of chemicals. Available at: http://www.unep.org/chemicalsandwaste/Portals/9/ Mainstreaming/CostOfInaction/Report_Cost_of_Inaction_Feb2013. pdf

Wang J, Eldridge M, Menn F-M, Dykes T, Sayler GS (2015a) Standardized application of yeast bioluminescent reporters as endocrine disruptor screen for comparative analysis of wastewater effluents from membrane bioreactor and traditional activated sludge. Ecotoxicology. doi:10.1007/s10646-015-1556-z

Wang Y-B, He C-G, Sheng L-X, Guo T, Wang X-F (2015b) Towards multi-level biomonitoring of nematodes to assess risk of nitrogen and phosphorus pollution in Jinchuan wetland of Northeast China. Ecotoxicology 24:1-10

Wu J, Dai Y-R, Rui S-Y, Cui N-X, Zhong F, Cheng S-P (2015) Acclimation of Hydrilla verticillata to sediment anoxia in vegetation restoration in eutrophic waters. Ecotoxicology 24:1-9

Xing Y-N, Chen X-J, Zhuang J, Chen X (2015) What happens when pharmaceuticals meet colloids: a review. Ecotoxicology 24:1-15

Xiong L, Tong Z-H, Chen J-J, Li L-L, Yu H-Q (2015) Morphologydependent antimicrobial activity of $\mathrm{Cu} / \mathrm{Cu}_{\mathrm{x}} \mathrm{O}$ nanoparticles. Ecotoxicology 24:1-6

Xu X-H, Oliff K, Xu T-T, Ripp SA, Sayler GS, Zhuang J (2015a) Microbial availability of mercury: effective detection and organic ligand effect using a whole-cell bioluminescent bioreporter. Ecotoxicology 24:1-7

Xu T-T, Marr E, Lam H, Ripp SA, Sayler GS, Close D (2015b) Realtime toxicity and metabolic activity tracking of human cells exposed to Escherichia coli $\mathrm{O} 157: \mathrm{H} 7$ in a mixed consortia. Ecotoxicology 24:1-8

Yin J-B, Zhang X-X, Wu B, Xian Q-M (2015) Metagenomic insights into tetracycline effects on microbial community and antibiotic resistance of mice gut. Ecotoxicology 24:1-8 
Zeng X-F, Wang Z-W, Wang J, Guo J-T, Chen X-J, Zhuang J (2015) Health risk assessment of heavy metals via dietary intake of wheat grown in Tianjin sewage irrigation area. Ecotoxicology 24:1-10

Zheng G-D, Chen T-B, Yu J, Gao D, Shen Y-J, Liu H-T (2015a) Impacts of composting strategies on the degradation of nonylphenol in sewage sludge. Ecotoxicology 24:1-7
Zheng M-Z, He C-G, He Q (2015b) Fate of free chlorine in drinking water during distribution in premise plumbing. Ecotoxicology $24: 1-5$ 